stomatologie 2013 [Suppl 1] · 110:11-11 DOI 10.1007/s00715-013-0287-0

(c) Springer-Verlag Wien 2013

\title{
Grußworte des Präsidenten der Österreichischen Zahnärztekammer
}

150 Jahre sind fürwahr ein stolzes Alter für eine zahnärztliche Organisation wie die Österreichische Gesellschaft für Zahn-, Mund- und Kieferheilkunde (ÖGZMK). Der Vorläuferverein der ÖGZMK, der „Verein Österreichischer Zahnärzte" (VÖZ) wurde bereits 1861 von Moriz Heider ins Leben gerufen und erfreut sich seit dieser Zeit bis heute eines aktiven wissenschaftlichen Lebens.

Besonders eindrucksvoll wird diese lange Zeit dann, wenn man vergleicht, wie lang andere wichtige Einrichtungen im Gesundheitswesen existieren:

Erste Krankenkassen gab es ab 1889, Ärztekammern seit 1891, den Hauptverband der Sozialversicherungsträger seit 1949 und das Gesundheitsministerium wurde - sieht man von einem kurzen Intermezzo während des Ersten Weltkriegs ab - überhaupt erst 1972 gegründet.

$\mathrm{Ob}$ die angeführten Institutionen im Lauf ihres Bestands ebenso erfolgreich waren wie die ÖGZMK, mögen andere beurteilen, für die österreichischen Zahnärzte und auch für mich persönlich steht fest, dass es ein wesentliches Verdienst der ÖGZMK war, zu erreichen, dass die Zahnmedizin eine heute nicht mehr wegdenkbare anerkannte Spezialdisziplin der Humanmedizin darstellt - etwas, was in der Vergangenheit nicht immer selbstverständlich war.
Ein Berufsstand wird nur dann auf Dauer erfolgreich sein, wenn es gelingt, aus den Erfahrungen der Vergangenheit heraus für die Herausforderungen der $\mathrm{Zu}$ kunft gewappnet zu sein. Dass zu diesem Vorhaben vor allem die Weiterentwicklung im wissenschaftlichen Bereich gehört, ist wohl unumstritten, genauso wie die Tatsache, dass die ÖGZMK dabei die unverzichtbare Institution für alle Zahnärzte ist. Deshalb freut es mich besonders, dass es gerade in den letzten Jahren gelungen ist, früher vereinzelt bestehende Meinungsunterschiede zwischen Standesvertretung und wissenschaftlicher Gesellschaft zu beseitigen und zu erreichen, dass die beiden wichtigsten zahnärztlichen Organisationen an einem Strang ziehen!

Ich darf in diesem Sinn der ÖGZMK die besten Wünsche der österreichischen Zahnärzteschaft zu diesem besonderen Jubiläum übermitteln und dies mit dem Wunsch auf eine ebenso erfolgreiche $\mathrm{Zu}$ kunft verbinden.

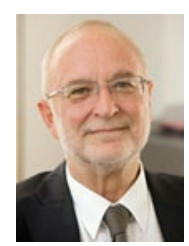

OMR DDr. Hannes Westermayer Präsident der Österreichischen Zahnärztekammer 